$$
666.911 .004 .12=60
$$

$684 \cdot 43$

\title{
influencia que ejerce el hidróxido cálcico del cemento y su evolución durante los fenómenos de la hidratación, sobre la resistencia de morteros y hormigones expuestos a la corrosión
}

\author{
F. W, MEIER-GROLMAN
}

Tübingen

\section{Introducción}

La producción mundial de cemento (230 millones de tonelados anualest "ll y el hecho de que las investigaciones sobre construcciones de hormigón en puentes, pistos $y$ construcciones hidráulicas, señalen como causa de la destrucción del hormigón la lixiviación y la influencia de las sales, hocen del problemo de la resistencia de los morteros y hormigones contra la agresión una de las cuestiones fundamentales más importantes y actuales de la corrosión. Aunque este problema está conocido ya desde el principio del siglo en toda su profundidad y las investigaciones científicas de la constitución, hidratación y propiedades del cemento han hecho grandes progresos en los últimos decenios, el hecho de que el hormigón acobado sufre influencias posteriores, que no se pueden evitar con los procedimientos tradicionales de fabricación de hormigón, en aguas y terrenos nocivos '2! , es la prueba de que muchos problemas no se han resuelto hasta ahora.

\section{I}

A causa de la einaccesibilidad" experimental y onalítica del hormigón endurecido. la investigación sobre las causas y el curso de la agresión del hormigón se encuentra con muchas dificultades, La teoría capilar, aplicada primero como hipótesis de trabajo, llevó al autor, en la valoración de los ensayos de conservación a largo plazo del hormigón en oguas naturales que contienen sulfatos y $\mathrm{CO}_{2 r}$ a nuevas opiniones teóricas sobre los procesos de la hidratación y del endurecimiento del mortero, que se desvían en puntos importantes de los conceptos aceptados hasta ohora sobre estos procesos y su importancia para apreciar las causas y el curso de la agresión ${ }^{(3)}$.

\subsection{Consideraciones teóricas sobre el cemento y los capilares}

Puesto que estas consideraciones son de importancia fundamental como puntos de fartida para la solución del problema de la resistencia del hormigón contra la agresión, es preciso hacer primero un resumen de lo más importonte:

En el endurecimiento hidráulico del cemenfo portlond mezclado con agua, considerado como un sistema compuesto por una fase sólida y una líquida, sólo el $40 \%$ del aguo se fija químıcamente durante el proceso de hidratación, lo que produce una reducción del volumen total de las fases sólida $y$ líquida $y$, por tanto, uno contracción, o la cual el sistema endurecido sólo puedé ceder en uno medida limitada, $y$, por ello, en el cemento endurecido nacen poros. Parte del agua de cmasado se fija entre las partículas de los nuevos compuestos, como película seudo-sólida enlazada a las moléculas por medio de fuerzas copilares, mientros el resto rellena los poros capilares como agua libre, que se puede evaporar y que contiene los componentes disueltos durante la hidra- 
tación en la fase líquida. Por eso no hoy ningún hormigón totalmente impermeable, $p$ sesto que el gel del cemento endurecido, semejante ol gel de sílice, formo un sistema de poros finísimos, atravesodo por una red más grueso de poros capilares, que se originan dentro del aglomerante endurecido como consecuencia inmediata del proceso de hidrotación.

Según estas consideraciones teórico-capilares, la actividad de la corrosión depen. de, en el primer período del ataque, de la cantidad de componentes del cemento portland disueltos en la fase líquida y que se depositan en los poros capilares de la pasta del mortero. Por eso reaccionan con los medios agresivos sólo los álcalis, el hidróxido cálcico liberado, los hidratos del $\mathrm{C}_{3} A$ y sus-productos de reacción; mientras las nuevas formaciones que proceden de la hidratación en la fase sólida lespeciolmente el hidróxido cálcico formado topoquímicamente en lo hidratación del $\mathrm{C}_{3} \mathrm{~S}$, del $\mathrm{C}_{2} \mathrm{~S}$ y del $\mathrm{C}_{4} \mathrm{FAl}$ se depositan en los poros del gei y quedan protegidos por la masa del gel silicico poco soluble.

Los procesos de agresión se desarrollarán, por esto, primero exclusivamente en los espacios capilares, puntos de menor resistencia $y$, por eso, es de la mayor importancia el que también allí tenga lugar la cristalización, disolución y recristalización de los nuevos compuestos como consecuencia de las influencias agresivas.

Las mismas consideraciones valen para los aglomerantes de mezclo, como los cementos ferro-portland, de Trass, de altos hornos y las mezclas de cemento portland con puzolana natural o artificial, puesto que en éstos empiezan los procesos de endurecimiento y de hidratación del clínker de portland - como en el cemento portland puro-antes de que comiencen las actividades reciprocas entre éste y los materiales adicionados, los cuales tienen influencia sólo en las fases posteriores del endurecimiento. Como consecuencia, el valor de una adición dependo, en primer lugar, de su capacidad para fijar los componentes del clínker en los poros capilares envolviéndolos o protegiéndolos $\mathrm{y}$, también, de la resistencia que los productos de reacción posean contra un ambiente constantemente agresivo.

\subsection{Los fenómenos del ataque en la pasta de mortero según las nuevas opiniones}

Según estas opiniones son de esperar las tres fases siguientes durante el ataque en la pasta de mortero:

1) Un afaque relativamente rópido, caracterizodo por la disolución o transformación del hidróxido cálcico existente en los poros capilores, originado por el CaO libre en el aglomerante.

2) Una reacción entre el medio agresivo y los hidratos poco estables del $C_{3} A$ o los hidratos del sulfoaluminato.

3) Un proceso mucho más lento, posterior a estas reacciones primarias que son relotivamente rápidos, de atoque continuo a la masa, de poros finisimos, de gel silícico del cemento. Este proceso se caracteriza por la descomposición de los hidratos de los silicatos cálcicos con extracción de hidróxido cálcico formado en las reacciones topoquímicas, hasta destrucción total del hormigón.

Se opina que los procecos 11 y 2) difieren claramente en las velocidades de reacción, según la energía del medio agresivo, - que transcurren más o menos uno en el otro. La fase 3 de agresión, sin embargo, debe, si las teorías del cemento son correctas, caracterizarse por un cambio marcado de la velocidad de agresión comparada con las fases 1 ) y 2 l.

\section{Las causas y el desarrollo del afaque en la pasta de mortero}

\section{I. Ensayos a largo plazo, su va- lor y significación}

Para la investigación y la evaluación de la dependencia entre las causas y el curso del ataque, primero se valoraron los resultados de los ensayos a largo plazo con probetas definidas de hormigón, mediante la diferencia de las resistencias, en " $/ \mathrm{m}$, después de conservados en agua y en materiales agresivos. Si se acepta como normal el aumento de la resistenciá después de conservadas en agua corriente, la diferencia 
de resistencia, en $\%$, es un valor absoluto para la medida del ataque, y se puede expresar gráficamente en función del tiempo durante el cual el hormigón permanece en el agua. Si el incremento del atoque es lineal, la velocidad se puede expresar por el coeficiente angular de las curvas resistencia-tantos por ciento entre los diversos ensayos. Así, se pueden expresar estas relaciones en cifras y gráficamente.

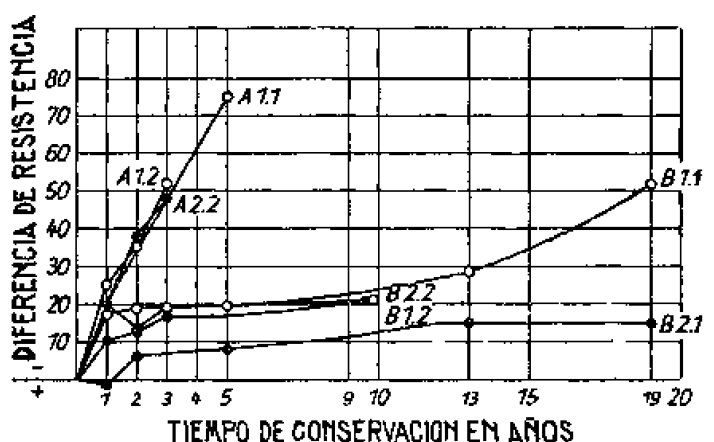

Fig. 1.-Diferencios de resistencia mecónicas, en $\%_{r}$ del mortero $y$ del hormigón de cemento portland fipo 225 , y de cemento de altos hornos, tipo 225 , en proberas conservados agresividad. Tiempo de conservación en años.

$\longrightarrow$ cemento portand $=1$.

$\mathrm{A}_{1}=$ Lago de Medicine en los EE. UU.

$B_{1}=$ Agua de mar (Mar del Norte)

$\mathrm{A}_{x}=$ Aguo cenagosa corriente de Berrau (Máx. $97 \mathrm{mg}$ de $\left.\mathrm{CO}_{3} / \mathrm{l}\right)$ ).

$\mathrm{B}_{2}=$ Agua cenagosa estancada de Bernou (Mâx. $97 \mathrm{mg}$ de $\mathrm{CO}_{2}[$ ).

La figura 1 representa la diferencia de resistencias mecánicas, en $\%$, del mortero de cemento y del hormigón fabricodo con cemento portland y con cemento de hornos altos $1350 \mathrm{~kg}$ y $400 \mathrm{~kg}$ de cemento $/ \mathrm{cm}^{3}$ : factor agua/cemento $=0,45$, en probetas con. servadas bajo diferentes aguas naturales que contienen $\mathrm{CO}_{\mathrm{y}}$ y sulfoto, en función del tiempo de conservación expresado en años. La línea $A$ expresa gráficomente las alteraciones de la resistencia (en $\%_{0}$ ) bojo la influencia del agua del Logo «Medicines en los EE. UU. ${ }^{41} y$ en agua cenagoso fluyente, que contiene, como máximo, $97 \mathrm{mg}$ de $\mathrm{CO}_{2} / 1^{(5)}$, la curva $B$ representa iguales magnitudes en el agua del Mar del Norte ${ }^{|6|}$ $y$ en agua cenagosa estancada. El Lago "Medicine" tiene un contenido de sulfato tres veces mayor que el agua del Mar del Norte.
Lo gráfico revela lo siguiente:

En los dos casos, $A$ y $B$, el hormigón de los dos tipos sufre una reacción primaria inmediato entre 1 y 1,5 años. Las diferencias de la resistencia porcentual se encuentran en una estrecha zona de dispersión. En $A$, el cemento portland $y$ el cemento de altos hornos no se distinguen. Mientros en $A$ no se notan diferencias importantes en las curvas de resistencia, porque la reacción primaria progresa más rópidamente debido a la fuerte influencio, en $B$, por el contrario, se nota una clara diferencia. En $B$ empieza, después de la reacción primaria, uno fase de cgresión constante hasta los 5 años, causada por la precipitación de hidróxido de magnesio al reaccionar el sulfato de magnesia con el hidróxido del calcio y, también, por la acción del $\mathrm{H}_{2} \mathrm{CO}_{3}$, en las aguas cenagosas estancadas, con formación de carbonato de calcio. Después de este período, el hormigón de cemento portland sufre el ataque del agua del mar, despacio primero $y$ después con velocidad creciente hosta la destrucción total; mientras en el hormigón de alto horno, la agresión sólo progresa lentamente y hasta se detiene. En el agua cenogosa estancada, la agresión de los dos tipos de hormigón se detiene bastante pronto, a cousa, evidentemente, de un equilibrio naciente entre el $\mathrm{CO}_{2}$ agresivo y los bicarbonatos, pero se nota una diferencia marcada en la magnitud de la reacción primaria entre los cementos portland y el de alto horno.

El ángulo $\propto$, que expresa la velocidad de ogresión en función del tiempo de conservación, Fig. 1, revela claramente la reacción primaria común a los diversos tipos de hormigón. Es notable que, bojo la fuerte acción del agua cenagosa fluyente, el ataque del cemento portlond continúa después de terminada la reacción primaria; mientras en el cemento de alto horno, se nota entre $1 / 2$ y 1 año una disminución de la velocidad de atoque.

La comparación de las diferencias de resistencios, en ${ }^{\circ}, 0, y$ el ángulo $\propto$, en los ensayos a largo plazo, puso de mánifiesto la existencia de una reocción primaria inmediata en el hormigón de oltos hornos y de cemento portland, debida, según la teoría capilar del cemento, a las fases de agresión 1) y 2). 


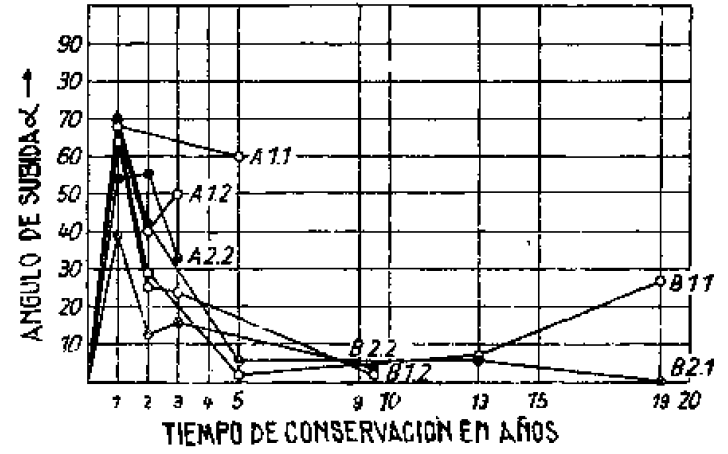

Fig. 2. - Valores del coeficiente angular de las curvas representadas en la figura 1. Tiempos de conservación en años.

$\longrightarrow$ cemento portland $=1$

cemento de altos hornos $=2$

Angulo $a 0$ a $20^{\circ}$ crecimiento muy lento

20 a $40^{\circ}$ crecimiento lento

40 a $60^{\circ}$ crecimiento rápido

60 a $80^{\circ}$ crecimiento muy rápido

\subsection{Evaluación y significación analítica de la cal extrafda por lixiviación}

Las causas y el desarrollo de la reacción primaria de ataque se pueden reconocer mediante determinaciones analíticas de lo col existente en el líquido de conservación del hormigón, si este agua es corriente y adicionada de $\mathrm{CO}_{2}$, puesto que así no existen tronsformaciones que enmascaren los resultados. Estos se expresan en $\%$ CaO referido al peso de cemento y an gramos de $\mathrm{CaO} / 1^{|p|}$. Los resultados coinciden con las investigaciones realizadas en otros lugares, sobre la extracción de la cal sacada de probetas de hormigón de alto horno 19.

La figura 3 expresa la cal extraída, en $\%$ referido al peso de aglomerantes $y$ en $\mathrm{g} \mathrm{CaO} / 1$, de prismas de $5,5 \times 5,5 \times 20 \mathrm{~cm}$ obtenidos de tubos de hormigón cuya dosificación es $380 \mathrm{~kg}$ de cemento por $\mathrm{m}^{3}$; tamaño de los áridos: 0 a $15 \mathrm{~mm}$; aglomerante: $c \theta-$ mento portland y cemento de alto horno conservados en agua corriente con adición de $\mathrm{CO}_{2}$ irenovada cada i5 diasl, en dependencia del tiempo de conservación expresado en meses.

Podemos ver en las curvas del contenido de $\mathrm{CaO}$, en $\%$, que el hormigón de cemento portland y cemento de alto horno sufren iguales reacciones primarias, coincidiendo con las nuevas opiniones teóricas sobre el cemento. Se pueden reconocer 3 fases diferenciadas de agresión en la reacción primaria: Una reacción inmediata, hasta 1 mes. La extracción de $\mathrm{CaO}$, en $\%$, es de 1 a $1,2 \%$ lo cual corresponde al promedio del contenido de CaO libre en el cemento portland $y$ en el clínker, que es de $1,1 \%$. Después se verifica una segunda reacción, que tiene lugar hasta después de 5 meses y que se acerca asintóticamente a la curva de la tercer fase de agresión. La extracción total del $\mathrm{CaO}$, en $\%$, es de $4,2 \%$. Puesto que en las investigaciones correspondientes a probetas de hormigón ferroportland, cemento Trass $30 / 70$ y mezcias de

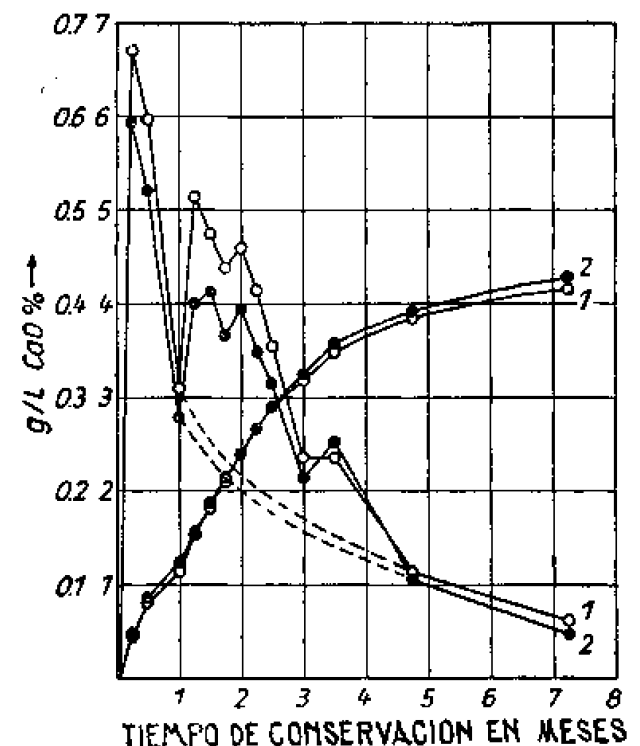

Fig. 3.-Extracción de cal, en ${ }^{v_{i}}$. CaO/peso de cemento y $\mathrm{g} \mathrm{CaO} / 1$, de prismas de $5,5 \times 5,5 \times 20 \mathrm{~cm}$ sacados de tubos fabricados con hormigon de cemento port and a cementa de allos hornos, conservados conservación en meses.

$\longrightarrow 0=$ cemento portland $=1$

= cemento de altos hornos $=2$

cemento portland y puzolana natural y artificial 1300 a $380 \mathrm{~kg} / \mathrm{m}^{2}$ contenido de cementol resultó un valor medio de 4,5\% de $\mathrm{CaO} /$ peso de cemento, se puede calcular que el valor capilar de agresión de la reacción primaria, es, en el mortero, 4 a $4,5 \%$ CaOlpeso de cemento. 
La gráfica de la extracción de cal lg $\mathrm{CaO} / 1)$ caracteriza las diversas fases de agresión y su curso.

No sólo los cementos normalizados, sino también cementos especiales pobres de $\mathrm{C}_{3} \mathrm{~A}$ (tipo ASTM V, contenido de $\mathrm{C}_{3} \mathrm{~A}<5 \%$ sufren, según la evaluación por medio de la diferencia de la resistencia en $\%$, en ensayos a largo plazo (conservación de 5 años en el lago de «Medicine»| ${ }^{(4)}$, una agresión primaria. Para los cementos especiales que no contienen ningún $C_{3} A$, se puede imaginar un curso de agresión primario como el de la línea de trazos en $\mathrm{g} \mathrm{CaO} / 1$.

\section{II}

\subsection{Combinación, resistente a la agresión, del hidróxido cál. cico del cemento, mediante transformaciones químicas durante la hidratación}

Las nuevas opiniones sobre los procesos de hidratación y de endurecimiento del mortero, sólo tendrían importancia teórica para aclarar las causas del ataque si no hubieran conducido a medidas prácticas para incrementar la resistencia contra la agresión.

Se recomendaba, hasta ahora, para evitar la reacción primaria en los capilares durante la hidratación, la fijación del hidróxido cálcico fácilmente soluble en la fase líquida, por medio de una reacción química, con objeto de reducir así considerablemente la agresión e incluso suprimirla.

El autor se ocupa en esta cuestión desde el año 1930. El problema es muy difícil, porque todos los materiales químicos necesarios para esta transformación - cuando se añaden al agua de amasado - tienen una influencia desfavorable en el proceso de fraguado y endurecimiento del mortero y del hormigón. Sólo la selección de materiales químicos, fijadores de cal, haría posible determinar su dosificación y sus reacciones en la hidratación y en el endurecimiento del cemento, y podría tener solución esta cuestión. Para esto, los fluoruros silícicos (fluatos) en una solución con agua resultaron muy útiles. La figura 4 son prismas de $5 \times 5 \times 15 \mathrm{~cm}$ de hormigón de alto horno,

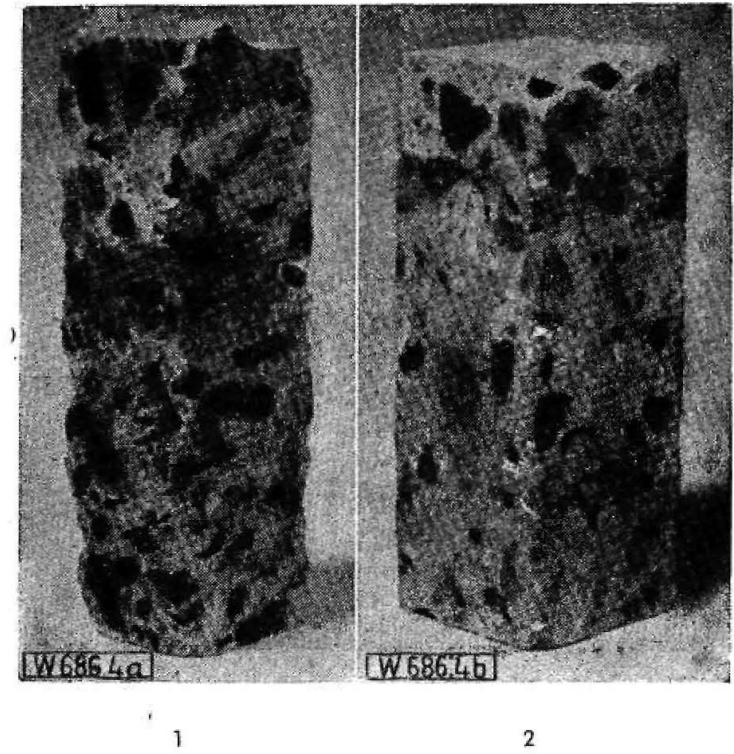

Fig. 4+-Prismas de $5 \times 5 \times 15 \mathrm{~cm}$ de hormigón de cemento de altos hornos $\left(225 \mathrm{~kg}\right.$ de cemento, $35 \mathrm{~kg}$ de Trass $^{2} \mathrm{~m}^{3}$ factor agua-cemento $=0,655$ ) despues de 1,5 años de conservación en agua destilada saturada con $\mathrm{CO}_{3}$ $\mathrm{pH}=3,5)$, sin y con la adición de fluato.

$1=$ hormigón sin adición; 2 = hormigón con adición al agua de amasado de $0,3 \%$ de fluato por peso de cemento.

los cuales se inmunizaron totalmente al impedir la reacción primaria por adición de fluato (sólo $0,3 \%$ peso de cemento), sumergidos en agua destilada estancada saturada con $\mathrm{CO}_{2}(\mathrm{pH}=3,5)$ durante más de 1,5 años. En el hormigón de cemento portland, la agresión se retarda mucho por la adición de $0,5 \%$ de fluato referido al peso del cemento. Los ensayos continúan ${ }^{19}$.

\subsection{Prueba de la unión del hidróxido cálcico del cemento, por trans- formaciones químicas durante la hidratación}

Era muy difícil probar el porqué y de qué modo tan pequeñas cantidades de elementos podían tener tal efecto, ya que, hasta ahora, no se suponía ningún efécto sobre las cantidades de hidróxido cálcico libre (el $11 \%$ ) contenidas en el hormigón endurecido ${ }^{103}(111) y$, por eso, era difícil comprobar las teorías y las experiencias a largo plazo. 


\subsubsection{Prueba por medio de in- vestigaciones analifticas}

Según el método con Glycolato ISchläepfer y Bukowskil no se podia demostrar, en trozos molidos de mortero normalizado, que existiese una combinación del hidrato de cal por sólo la adiciợ de fluato. Tampoco las medidas de la concentración de iones hidrógeno dieron resultado. En cambio sí se podía demostrar, por medio de la valoración conductimétrica de fluoruros compuestos, propuesta por Allenstein $y$ Kampmann [I2], que el mortero normalizado de cemento portland con $0,5 \%, 1 \%$ y $5 \%$ de fluato adicional, referido al peso de cemento, no contiene yo ningún flúor. Con este método indirecto se sacó la prueba analítica de la existencia de una reacción entre el fluato y la cal en el mortero.

Las medidas volumenométricas y las investigaciones con el microscopio electrónico han contribuído mucho a la aclaración del curso de hidratación en el cemento portland y en el cemento de alto horno, con y sin la adición de fluato.

\subsubsection{Prueba por medio de medi- das volumenométricas}

Con ayuda del volumenómetro de R. Dittrich ${ }^{1131}$ puede investigarse el cambio de volumen de los aglomerantes hidráulicos, después de mezclarlos con agua con una exactitud de $1: 50.000$, y se pueden examinar los materiales adicionales en cuanto a sus efectos sobre los cambios de volumen.

Las figuras 5 y 6 corresponden a los resultados de los ensayos volumenométricos realizados con cemento Portland 225, de la fábrica de Leimen, y cemento de alto horno 225 , de la fábrica de Rheinhausen icomo ejemplo para los dos tipos de cementol en función del tiempo y de la temperatura, con y sin la adición de fluato ${ }^{[14]}$.

El Raiasil BS es una combinación de fluato que se emplea desde el año 1949 y que estó proyectada para emplearla en la hidratación del cemento.

La curva de cemento portland, con y sin adición de fluato, permite deducir, claramente, las siguientes relaciones: Aparece un curso lineal hasta las $14 \mathrm{hr}$ de la hidratación del cemento portland a $20^{\circ} \mathrm{C}$, inflúdo, evidentemente, por la reacción inmediata idespués de amasar) del yeso con los elementos del cemento. Esta hipótesis queda iustificado según los resultados de las me-

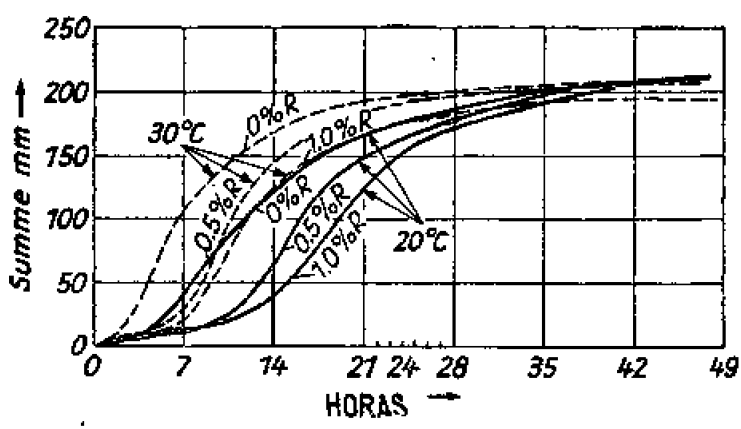

Fig. 5.-Representación gráfico de lo contracción len $\mathrm{mm} / \mathrm{sumg}$ en el volumenómetro de R. Dittrichl, del cemsnto portland
tipo 225 . Fóbrié Leimen, sin y con la adicíon de Rajasil BS, en Función del tiempo desde 0 hasta 48 horas y a las temperaturas de $20^{\circ}$ y $30^{\circ} \mathrm{C}$

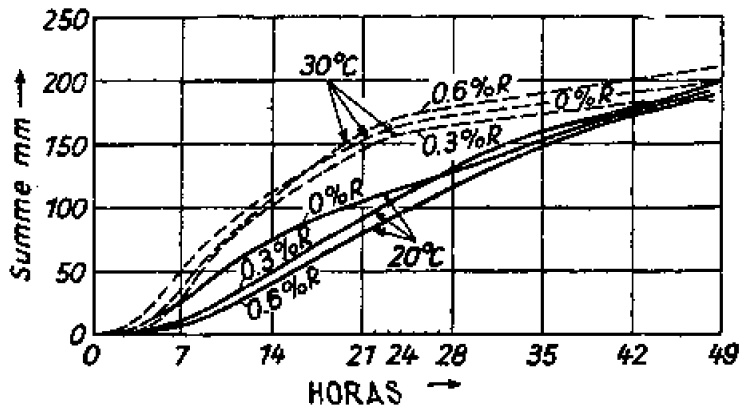

Fig. 6,-Representación gráfica de la contracción len $\mathrm{mm} /$ sume en el volu menómetro de $R$. Dittrich), del cementa de alto hernos ripo 225, Fábrica Rheinhousen, sin y con Rajasi temperaluras de $20^{\circ}$ y $30^{\circ} \mathrm{C}$. desde 0 hasta 48 hotas y a las

didas de contracción de volumen del cemento portland, con y sin yeso. Según la forma de la curva, la reacción del yeso fermina después' de 14 horas $y$, desde este momento, la contracción del volumen se acerca constantemente, y finalmente asintóticamente, hacia la constancio de volumen. La comparación de las curvas muestra que la reacción del yeso aumenta por la presencia de fluatos en la fase liquida, y que 
las reacciones de los fluatos se desarrollan en general simultáneamente junto con las del yeso.

Por los resultados de la figura 6 , se puede opreciar que en el cemento de alto horno se cumplen los mismas reacciones en el clínker que contiene este aglomerante.

\subsubsection{Crecimiento de las resistencias en los cementos ensayados}

Puesto que la acción de un material quimico es de gron importancia para el desarrollo de la resistencia en teoria y en la práctico, los cementos ensoyodos se exami-

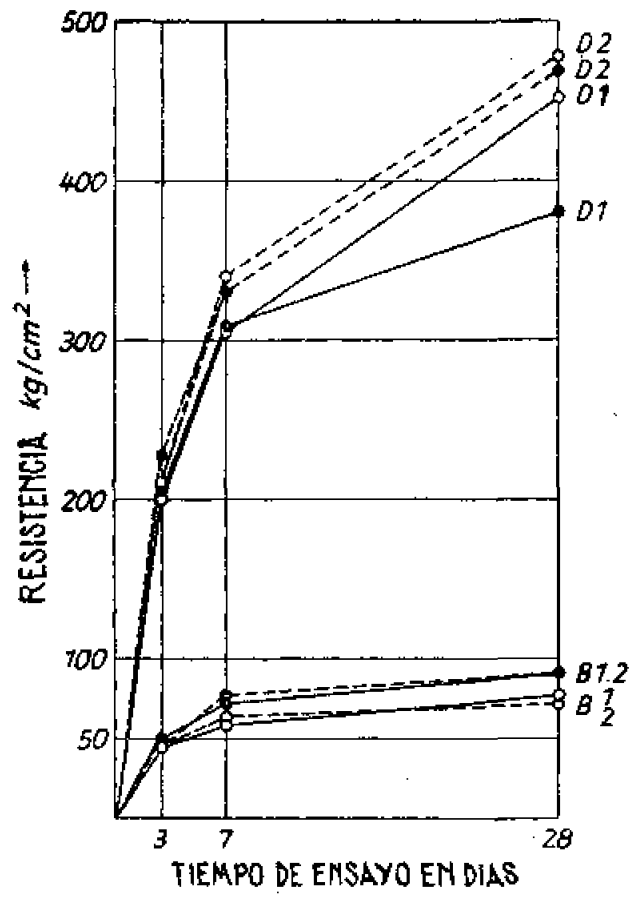

Fig. 7.-Resistenclas a la compresión, y flexo-tracción, según DIN 1164, de cemento portland tipo 225, Heiderberg, y de cemento de altos hornos tipo 225, Rheinhtusen, sin y con

-O = cemento Portland.

= cementa de altos hornos.

$=1=$ resistencia sin adición.

$=2=$ resistencia con adición de

Huale (C.P. $=\uparrow \%, \mathrm{CAH}=0,3 \mathrm{~h} / \mathrm{p}, c)$.

$B=$ resistencia a la flexotracción.

$D=$ resistencia a la compresión. naron, en lo referente a su resistencia a la compresión y a la tracción, según DIN 1164.

Vemos que los cementos desorrollan igualmente sus resistencias hasta un plazo de 7 días, de manera que se puedan comparar exactomente los resultados. El resultado después de 28 días, muestra que, por la adición de fluato, se obtiene un aumento de resistencia a la compresión en las dos especies de cemento, mientras la resistencia a la tracción y a la flexión no sufre ningún combio. En los ensayos con cubos de hormigón de cemento portland y de cemento de alto horno, según DIN 1048, la influencia de la presencia del fluato en el crecimiento de la resistencia se revela más clara aún; la resistencia a la compresión aumenta el 20 hasta el $30 \%$ y $10 \%$, respectivamente, y la resistencia a la tracción y flexión aumenta, después de 28 días, el $10 \%$ o el $5 \%$, también respectivamente. En el hormigón de cemento portland, la resistencia que se desorrolla al principio disminuye claramente a las 15,24 y 48 horas, por la adición del fluato, de acuerdo con los resultados volvmenométricos; después de 3 y 7 días, la disminución de la resistencia se recupera. Según los valores experimentales generales de la resistencia a la tracción y a la compresion, las partículas coloidales muestran buena resistencia a la tracción, mientros los enlaces cristalinos condicionan la buena resistencia a la compresión; de aquí, se debe sacar la conclusión de que se forman compuestos cristalinos por la adición del fluato.

\subsubsection{Investigaciones por me- dio del examen con ol microscopio electrónico}

Las investigaciones con el microscopio electrónico de las diversas fases de hidratación se hicieron después de 0-14.24 hr y 7 días. Los cementos amasados con agua destilada, con y sin adición de fluato, se depositaron, con $1 \mathrm{~mm}$ de espesor, en platinas, que se conservaron en desecador con 93 a $95 \%$ de humedad relativa y a la temperatura normal de la habitación, hasta su examen, para imitar las condiciones de mortero. El examen se hizo, según lá técnica de G. Schimmel del Instituto Batfelle e.V. en Frankfurt/Main, en un microscopio 
electrónico I Siemens con 80 kV de tensión de radiación ${ }^{116)}$.

El Instituto Battelle opina que los cementos no han sido profundamente examinados con microscopios electrónicos. Y como no había ninguna literatura disponible, se precisaban amplias investigaciones previas con el fin de encontrar un método adecuado.

Las figuras 8 hasta 11 corresponden a fotografías electro-microscópicas de cemento portland 225, tipo Leimen, sin y con $1 \%$ de fluato, referido al peso de cemento amasado según las normas y aplicado en platinas conservadas en desecador a $93-95 \%$ de humedad relativa después de 0-14-24 hr y 7 días y con ampliación de 15.000 diámetros.

Los cementos se examinaron inmediatamente después de amasar $(\mathrm{pH}>12)$ y mostraron granos únicos y compuestos, pero no se pudo reconocer todavía la hidratación. La adición de fluato al agua de amasado $(\mathrm{pH}>12)$, muestra claramente diferencias en la estructura de la superficie de los granos de cemento. Esta observación es interesante, puesto que puede ser la explicación del hecho de que, en las investigaciones anteriores, en las cuales se añadieron can- tidades mucho mayores de materiales químicos al agua de amasado para la fijación de la cal, la reacción rápida en la superficie de los granos de cemento influía de manera desfavorable en el proceso del fraguado y de la resistencia.

Los cementos examinados después de 14 horas acusaron una hidratación avanzada. Se observaron pequeños cristales con forma de agujas hexagonales regulares, que son menos perfectas y peor formadas al adicionar fluato. Estos cristales se parecen mucho a los de tipo hexagonal del $\mathrm{C}_{3} \mathrm{~A}$. $\mathrm{CaSO}_{4} \cdot \mathrm{H}_{2} \mathrm{O} \cdot \mathrm{H}_{12}$.

Después de 24 horas de hidratación, se pueden distinguir dos formas de cristales: las agujas hexagonales y las de forma de erizos. Con la adición del fluato, se observan especialmente los cristales hexagonales, y apenas las agujas. Los grupos de agujas (erizos) tienen formas parecidas, en el hábito cristalino, al hidróxido cálcico, (18), y son una prueba de que la reacción con el yeso se ha verificado, después de 14 horas, de acuerdo con las investigaciones volumenométricas, y que el cemento portland, después de 24 horas, empieza a hidratarse con precipitación de hidróxido cálcico en

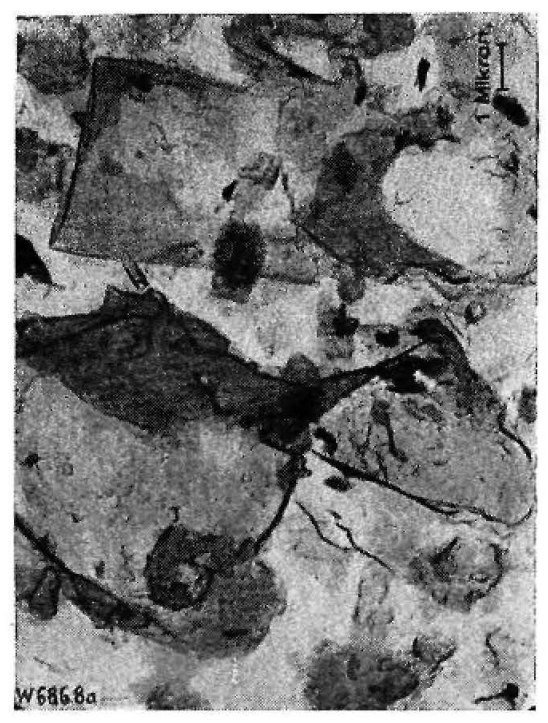

Sin

$\times 15.000$

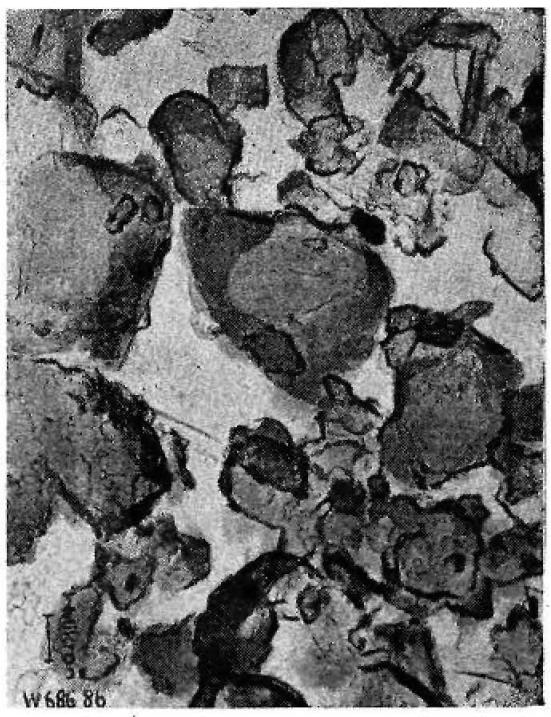

Con

Fig. 8.-Adición de fluato al cemento portland tipo 225, Fábrica de Leimen, y examinados inmediatamente después de amasados. 

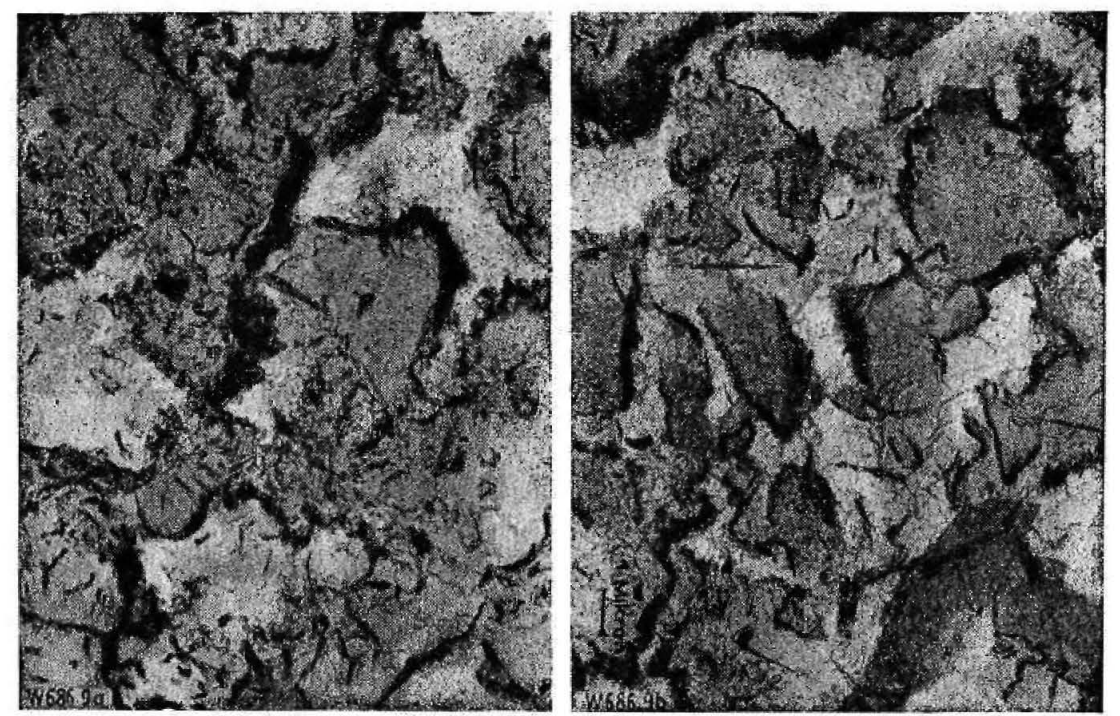

$\operatorname{Sin}$

$\times 15,000$

Con

Fig. 9.-Adición de fluato al cemento portland tipo 225, Fábrica de Leimen, y examinados 14 horas después de amasados.
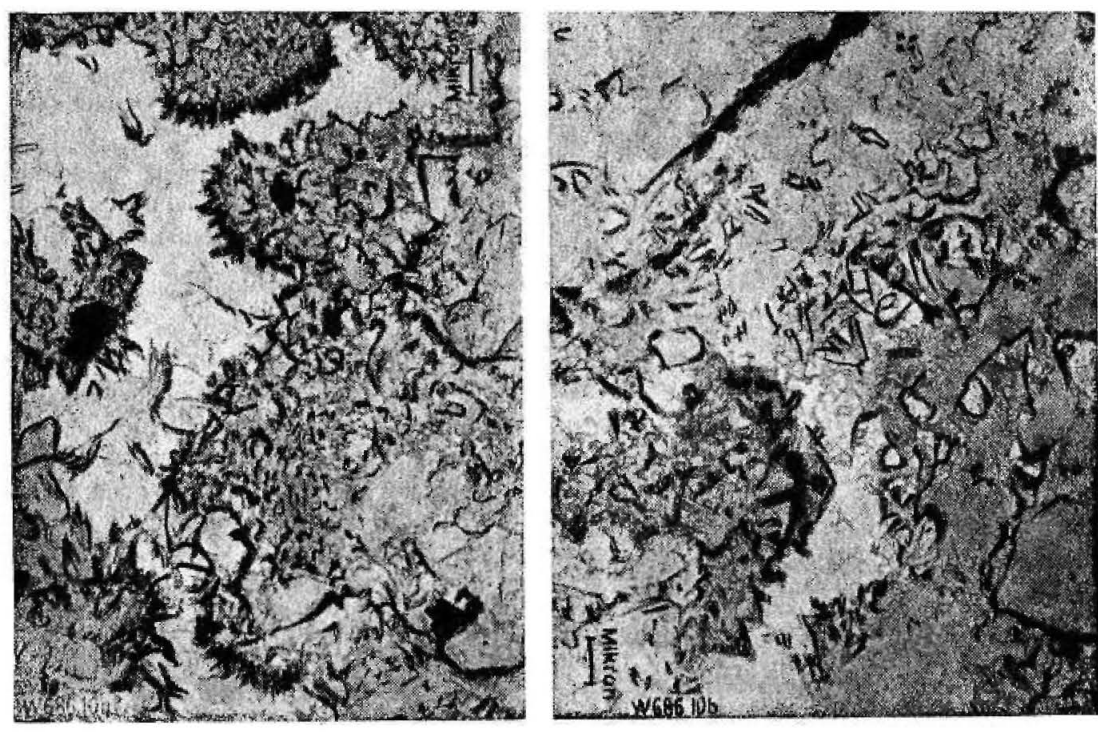

$\operatorname{Sin}$

$\times 15.000$

Con

Fig. 10-Adición de fluato al cemento portland tipo 225, Fábrica de Leimen, y examinados 24 horas después de amasados.

la fase líquida. Los cristales llamados «agujas» se forman, por el contrario, en la zona de contacto de las fases sólida y lí- quida, donde hay que suponer una mayor concentración de cal. Después de 7 días aparece una formación intensa de agujas 

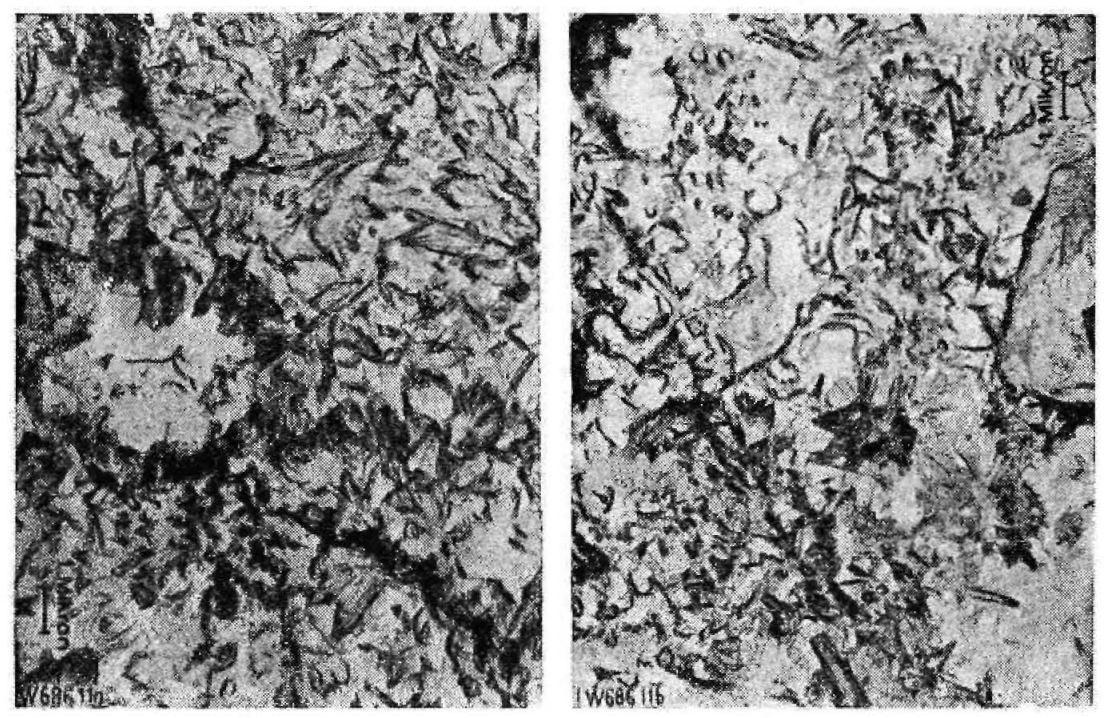

$\operatorname{Sin}$

$\times 15.000$

Con

Fig. 11.-Adición de fluato al cemento portland tipo 225, Fábrica de Leimen, y examinados 7 días después de amasados.

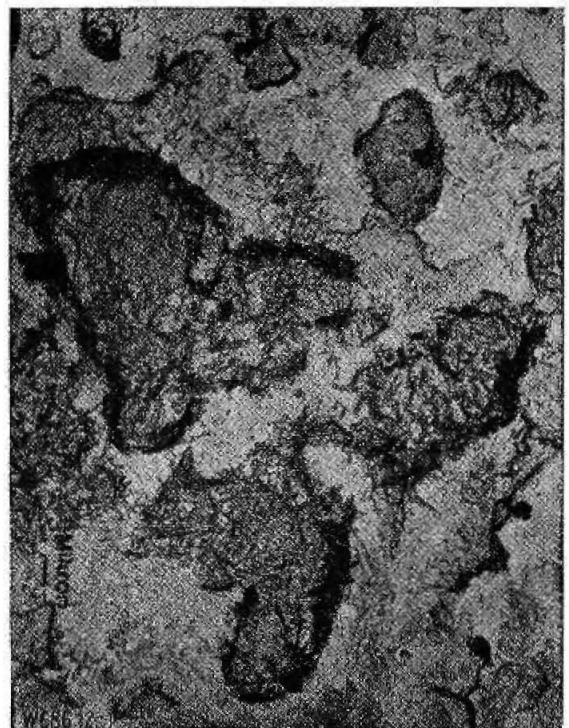

$\operatorname{Sin}$

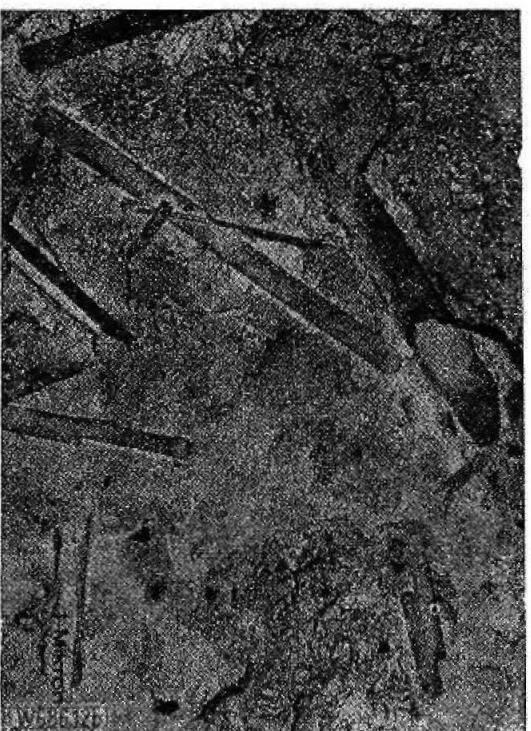

Con

Fig. 12.-Adición de fluato al cemento altos hornos tipo 225, Fábrica de Rheinhausen, y examinados 7 días después de amasados.

que rellena todo el espacio que ocupaba la fase líquida. Pero en el cemento con fluato, los espacios están rellenos con cristales tipo de tablillas hexágonas. Se puede observar claramente, en la preparación rápida, la protección de la superficie.

La figura 12 muestra una fotografía de microscopio electrónico de cemento de alto 
horno tipo 225, Fábrica Rheinhousen, sin y con $0,3 \%$ de fuato referido al peso de cemento, después de 7 días. Ampliación 8.400 diámetros.

Las fotografías después de 7 dias coinciden en el aspecto, con y sin adición de fluato. Al compararlas con las del cemento portland, se observa que en las foses de la hidratación del cemento de alto horno no hay ninguna formación de agujas. Esta observación es muy interesante y debe ser el punto de arranque para investigaciones posteriores.

Las fotografías microscópicas han mostrado la influencio de la adición del fuuafo en la hidratación del cemento. En la hidratación del cemento Portland, el fluato impide la formación de cristales (en forma de agujasl de hidróxido cálcico fácilmente solubles en los poros de cristal, evidentemente por la transformación de cristales hexagonales de forma de palitos, los cuales se distinguen, sin emborgo, por lo velocidad de formación, por el tamaño y por el aspecto de los cristales de $\mathrm{C}_{3} \mathrm{~A}$. $\mathrm{CaSO}_{4} \cdot \mathrm{H}_{12}$ y forman una capa protectora alrededor de la superficie de los granos de cemento por una reacción inmediata después de amasar el cemento, lo cual inicia una reacción topoquímica.

Las presentes investigaciones morfológicas no bastan para una interpretación quimica y cristalográfica exacta. Es de esperar que se obtendrán más resultados con fotografías de reflexión de electrones, sobre los compuestos formados en la hidratación y el endurecimiento de los dos tipos de cemento $y$ bajo la acción de materiales adicionoles que fijen la cal. Se han previsto investigaciones en medios agresivos.

No se puede emplear el estudio de A. Grudemo 117 sobre la mortología y las propiedades de cristalización de hidratos silícicos de la cal, para la evaluación de este material, puesto que exigen condiciones de preparación especiales. Sin embargo, se atribuye mucha importancio o los investigaciones del hidrato de la cal con el microscopio electrónico; estas investigaciones se hicieron según la iniciotiva de la Junta Federal de la Industria Alemana de la $\mathrm{Cal}{ }^{181}$ y los informes de $H$. zur Strossen en la conferencio de la Asociación de Fóbricos Alemanas de Cemento $1957^{\text {[19] }}$ sobre hidrogra- nates Icompuestos resistentes contra sulfatos, hidratos silícicos del aluminiol, que se forman al combinarse de 0,3 Mol. de $\mathrm{SiO}_{2}$ con los hidratos de $\mathrm{C}_{3} \mathrm{~A}$.

\section{Resumen}

La comparación de los ensayos a largo plazo, con los resultados analíticos y físicos del hormigón de cemento portland y de alto horno conservados en medios agresivos, permitió confirmar las nuevas hipótesis sobre el cemento en sus puntos más importantes, $y$, partiendo de ellas, se trató de aplicar los procesos de hidratación y de endurecimiento del mortero, osí como las causas y el curso de la agresión del hormigón.

Mediante la combinación, resistente al ataque, del hidróxido cálcico del cemento existente en la fase líquida, lograda durante la hidratación, se consiguió impedir y detener al ataque primario y dar al hormigón de cemento portland y de cemento de alto horno una resistencia química que, hasta ahora, no se habia conseguido.

Los resultados actuales, que se publicarán en breve en la Prensa científica ${ }^{20-211}$, abren nuevos caminos para la solución del problema de la ogresión del hormigón. No se puede esperar que, con esto sólo, todos los problemas tengan solución; pero a vista de las coincidencias alconzadas, según los diversos métodos de estudio, estas cuestiones alcanzan una importancia fundamental ${ }^{22 !}$.

\section{BIBLIOGRAFIA}

1. Producción mundial de cemento 1956; Zement-Kolk-Gips 10 (1957) 529.

2. DIN 1045 (1943), 1952; DIN 1047, 1943; DIN 1164 (1942), 1954; DIN 4030 i1930), 1954.

3. F. W. MEIER-GROLMAN: Sobre la importancia de la cal libre en el cemento Portland frente a la resistencia del hormigón contra los efectos agresivos; $\mathrm{Ze}$ ment-Kalk-Gips $9(1956) 15 / 28,58 / 71$. 
F. W. MEIER-GROLMAN: Influencia de la cal libre en el cemento Portland sobre la resistencia del hormigón frente a las acciones agresivas.

Instifuto Técnico de la Construcción y del Cemento, Monografía n. 182 (Madrid: 19571 .

4. D. G. MILLER y P. W. MANSON: Ensayos a largo plazo de hormigones $y$ morteros expuestos a la agresión de aguas sulfatadas.

Technical Bulletin 194, mayo 1951; Universidad de Minnesota, Estación experimental de Agriculturo.

5. BAYER. Landesanstait fūr Moorwirtschaff:

1. Informe de comprobación «Recientes ensayos sobre el comportamiento de diferentes mezclas de mortero y hormigón en el panfano Bernauers, del 26.12.1926. H. Dr. Schwaibold agradece al autor por el ofrecimiento de este informe.

2. Informe de comprobación del laboratorio técnico de la consfrucción de la Escuela Superior Técnica de München, del 4.10.1927 Zch. 510 y 31.1.1934 n.. 54 Zch. 1981. Este informe no aparecía ya existente como consecuencia de los efectos de la guerra. En esta ocasión el autor desea expresar su agradecimiento por la facilitación y amabilidad en la adquisición de estos documentos que han ofrecido los Sres. Prof. F. A. Finger, de la Escuela Superior de Arquifectura de Weimar, y el Director Franke, Ing. Dipl. del Instituto de Investigación de Materiales de Weimar.

6. A. ECKARDT y W. KRONSBEIN: Ensayos sobre el comportamiento del hormigón y cemento en agua de mar. Deutscher Ausschuss für Stahlbeton, H. 102 (Berlín: 1955).

7. Bayerische Landesgewerbeanstait MPA Nürnberg: Estudio de tubos de hormigón sobre la resistencia a la agresión. P. Ber. M-N. ${ }^{\circ} 27376119551$.

8. G. MALL: Estudios sobre el comportamiento del \&Thurament frente a las soluciones agresivas, Zement-Kalk-Gips 5
(1952) H. 12; parte de informe. En esta ocasión, el qutor desea expresar su agradecimiento al Sr. Dr. Ing. Mall por la facilitación de sus resultados sin publicor sobre el olmacenamiento de cementos de horno alto.

9. H. KORTE: Comprobación de Rajasil BS como producto adicional al hormigón, Baustoffprūfstelle des Wasser-und Schiffahrtsamtes, Neubauamt, MindenWeser, P. Ber. 1312-IVe del 26.3.56 P Ber. 3879-IVe del 21.9.1956 y 14.11.1957.

10. F. W. LOCHER: La cal libre del cemento $\mathrm{y}$ el efecto de producto adicional del hormigón Rajasil sobre la resistencia química del cemento; Zement-KalkGips 10 (1957) 221/31.

11.. F. W. MEIER-GROLMAN: Sobre la imporfancia de la cal libre y del aluminato cálcico en el cemento Portland para la resistencia del hormigón a los sulfatos; Zement-Kalk-Gips 10 \{1957) 231/8.

12. E. ALLENSTEIN: Laboratorio de química orgánica de la Escuela Superior de Stuttgart; informe personal, del 5.8.1955.

13. R. DITTRICH: $H .10$ del informe de la reunión de la industria del cemento (1954), S. 51/4, H. 12 (1956). K. Seidel: S. $151 / 71$.

Manual de la comprobación de materiales $2{ }^{\circ}$ edición tomo III (1957) S. 385 , 444.

14. Bundesanstalt für Strassenbou: Dept. de materiales: Comprobación de Rajasil BS como producto adicional para el hormigón. P. Ber. n. ${ }^{\circ} 11108$ del 8.7.1957 Abb. $2 / 4$ y $8 / 10$.

15. G. SCHRUBB: Comprobación del endurecimiento inicial del hormigón con adición de Rajasil BS. Laboratorio de Maferiales de la Construcción de Bayernwerk A. G., P. Ber. Lab. n. ${ }^{\circ} 5760 / 62$ del 6.11 .1956

16. G. SCHIMMEL: Estudio electromicroscópico sobre la influencia de la hidratación del cemento por Rajasi! BS. Battelle-Institut e.V. Frankfurt/M. P. Ber. Zch. $117-78 / \mathrm{K} / \mathrm{h}$ hel del 24.4 .1958 . 
17. A. GRUDEMO: Estudio electronográfico de la morfología y propiedades de cristalización de los hidratos de silicato de calcio. Handlingar n. $26 \quad$ (1955) Pp. 103. Svenska Forskningsin 'Stitutet för Cement och Betong Vid. Kunigl. Tekniska Högskolan I Stockholm.

18. G. SCHIMMEL: Pasta de cal en el microscopio electrónico; Zement-KalkGips 11 (1958) 46/9.

19. H. ZUR STRASSEN: Las reacciones quimicas en el endurecimiento del cemento; Zement-Kalk-Gips 11 (1958) 137/43.
20. F. W. MEIER-GROLMAN: Sobre las causas y el desarrollo de la agresión de aguas agresivas sobre el mortero de cemento o sobre el hormigón. Sin publicar.

21. F. W. MEIER-GROLMAN: La inmunización de morteros y hormigones frente al efecto de aguas agresivas por unión del hidrato de cal en el cemento en la hidratación por fluatos de composiciones especiales. Sin publicar.

22. R. H. BOGUE: Temas sobre la investigación del cemento. Conferencia de la reunión especial del Ver. Dtsch. Zementwerke 2.11.1956 en Düsseldorf. Zement-Kalk-Gips 10 (1957) 162/6.

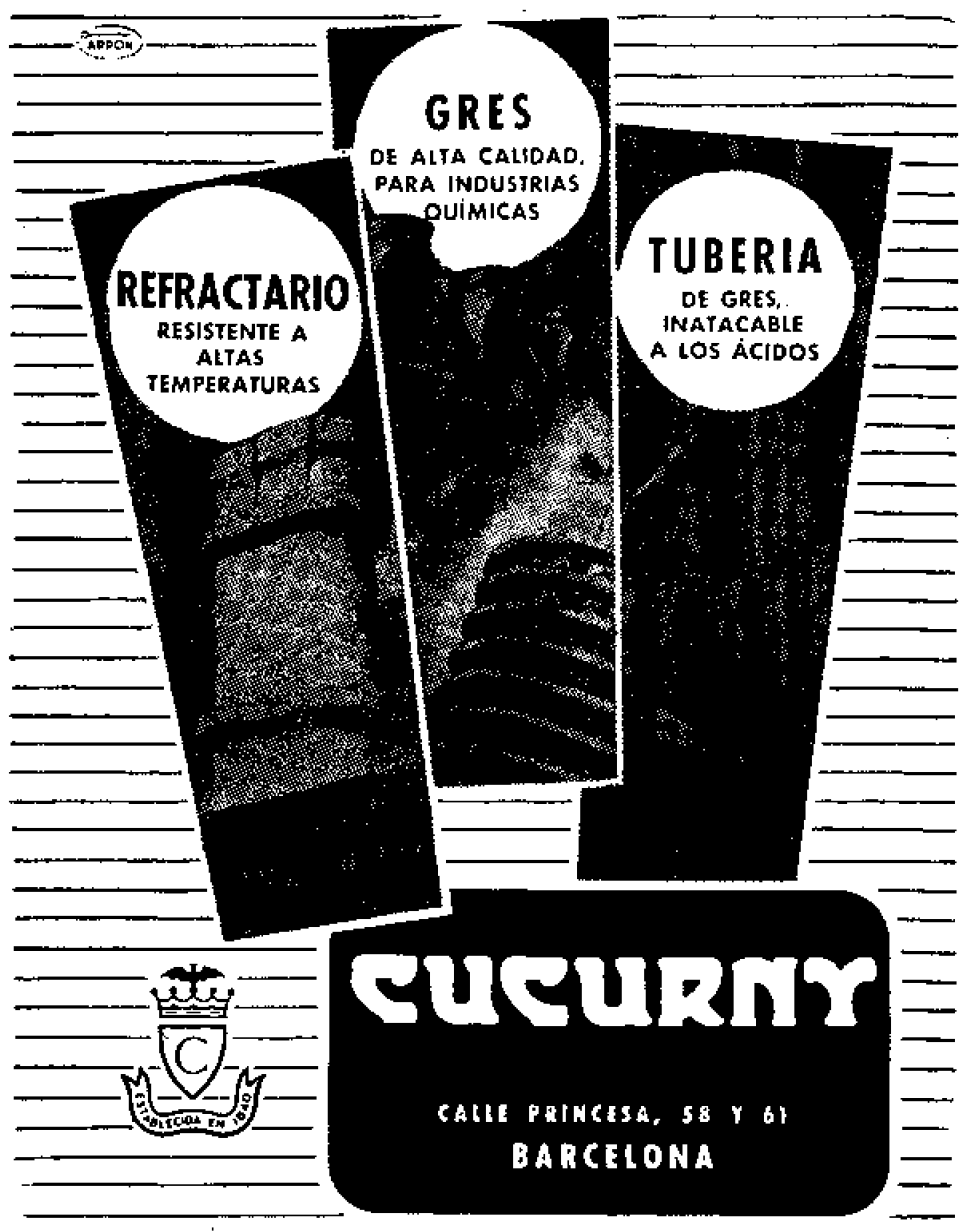

